\title{
Bacterial artificial chromosome derived simian varicella virus is pathogenic in vivo
}

\author{
Christine Meyer ${ }^{1}$, Jesse Dewane ${ }^{1}$, Kristen Haberthur ${ }^{2}$, Flora Engelmann ${ }^{4}$, Nicole Arnold ${ }^{4}$, Wayne Gray ${ }^{5}$ \\ and IIhem Messaoudi ${ }^{1,2,3,4,6^{*}}$
}

\begin{abstract}
Background: Varicella zoster virus (VZV) is a neurotropic alphaherpesvirus that infects humans and results in chickenpox and herpes zoster. A number of VZV genes remain functionally uncharacterized and since VZV is an obligate human pathogen, rigorous evaluation of VZV mutants in vivo remains challenging. Simian varicella virus (SW) is homologous to VZV and SW infection of rhesus macaques (RM) closely mimics VZV infection of humans. Recently the SW genome was cloned as a bacterial artificial chromosome (BAC) and BAC-derived SW displayed similar replication kinetics as wild-type (WT) SW in vitro.
\end{abstract}

Methods: RMs were infected with BAC-derived SW or WT SW at $4 \times 10^{5}$ PFU intrabronchially ( $N=8,4$ per group, sex and age matched). We collected whole blood (PBMC) and bronchoalveolar lavage (BAL) at various days postinfection (dpi) and sensory ganglia during latent infection (>84 dpi) at necropsy and compared disease progression, viral replication, immune response and the establishment of latency.

Results: Viral replication kinetics and magnitude in bronchoalveolar lavage cells and whole blood as well as rash severity and duration were similar in RMs infected with SW BAC or WT SW. Moreover, SW-specific B and T cell responses were comparable between BAC and WT-infected animals. Lastly, we measured viral DNA in sensory ganglia from both cohorts of infected RMs during latent infection.

Conclusions: SW BAC is as pathogenic and immunogenic as WT SW in vivo. Thus, the SW BAC genetic system combined with the rhesus macaque animal model can further our understanding of viral ORFs important for VZV pathogenesis and the development of second-generation vaccines.

Keywords: Herpesvirus, Simian varicella virus, Rhesus macaque, Bacterial artificial chromosome, Pathogenesis

\section{Introduction}

Varicella zoster virus (VZV) is a neurotropic alphaherpesvirus and the etiological agent of varicella (chickenpox) and herpes zoster (shingles). VZV establishes latency within the sensory ganglia, and reactivation from latency can cause significant morbidity and occasionally mortality in older and immunocompromised individuals. Currently the FDA vaccine Zostavax ${ }^{\oplus}$ reduces the incidence of shingles by $51 \%$ and the burden of disease by approximately $61 \%[1,2]$. Thus, a significant portion of vaccine recipients still remains susceptible to VZV

\footnotetext{
* Correspondence: messaoud@ucr.edu

'Vaccine and Gene Therapy Institute, Oregon National Primate Research Center, Beaverton, OR 97006, USA

${ }^{2}$ Molecular Microbiology and Immunology Department, Oregon National Primate Research Center, Beaverton, OR 97006, USA

Full list of author information is available at the end of the article
}

reactivation. To improve vaccine efficacy, we need to determine the function of the viral open reading frames (ORFs) that contribute to VZV pathogenesis and those that are important for the host immune response.

Simian varicella virus (SVV) is a homolog of VZV that causes varicella-like disease and establishes latency in sensory ganglia of rhesus macaques [3-5]. SVV shares significant DNA homology and genome colinearity with VZV [6-9]. VZV and SVV have the smallest genomes of the herpesvirus family. VZV encodes at least 70 unique ORFs and SVV encodes 69 distinct ORFs $[8,10]$. Despite the smaller genome size and homology to herpes simplex virus (HSV), a number of VZV/SVV genes remain functionally uncharacterized. Studies characterizing viral gene function utilizing in vitro tissue culture models do not always model the complex host-pathogen 
relationship that occurs in vivo. Recently, with the construction of an infectious SVV bacterial artificial chromosome (BAC), the production of mutations and deletions in specific SVV ORFs will allow the investigation of gene function during in vivo infection [11]. Previously, SVV BAC was shown to generate infectious virus with molecular properties and in vitro replication kinetics comparable to wild-type (WT) SVV [11]. SVV BAC was also used to generate an ORF10 deletion virus, which demonstrated that SVV ORF10 is nonessential for replication in vitro [11]. In the current study we further investigate SVV BAC in vivo by monitoring replication kinetics, immune response and establishment of latency in rhesus macaques and show that SVV BAC is as pathogenic as WT SVV.

\section{Results}

\section{Whole-genome analysis of SVV BAC}

The BAC derived SVV viral genome was comprehensively analyzed by comparative genomic hybridization (CGH) and directly compared to wild-type (WT) SVV. Using this technique, any differences in genomic sequence between SVV BAC and WT SVV results in variations in hybridization intensities to corresponding segments represented on the array, giving an altered hybridization ratio between SVV BAC and WT SVV (Figure 1A). CGH analysis revealed that two areas displayed variations when compared to WT SVV, indicating differences in nucleotide sequence at these locations. These regions were amplified via PCR and directly sequenced resulting in the identification of two nucleotide substitutions that produced 1 missense mutation and 1 silent mutation within the coding region of the SVV BAC genome. Specifically, we identified a point mutation at nucleotide 41990 from G to A within ORF22, producing an amino acid change from valine to isoleucine (Figure 1B) and a transition of nucleotide 106546 from $\mathrm{T}$ to $\mathrm{C}$ producing a silent mutation within ORF62/71 (Figure 1C). The nucleotide change in ORF62/71 was also previously shown in the sequencing of an ORF61 deletion virus that was generated from the same parental SVV cosmid system [11-13]. SVV ORF22 is a putative tegument protein based on the function of herpes simplex virus type-1 (HSV-1) UL36 homolog. The missense mutation in ORF22 did not render SVV BAC derived virus noninfectious or hamper replication kinetics and plaque size in vitro [11].

\section{Disease severity and viral load}

Rhesus macaques (RMs) were infected with SVV BAC or WT SVV at $4 \times 10^{5}$ PFU intrabronchially $(n=4$ per group, sex and age matched). We investigated the pathogenesis of BAC derived SVV in vivo by measuring disease progression, viral replication, immune response, and the establishment of latency compared to WT SVV. We collected bronchoalveolar lavage (BAL) cells and blood (peripheral blood mononuclear cells, PBMC) at various days post-infection (dpi) and sensory ganglia were collected at necropsy (84-86 dpi). All infected RMs displayed hallmarks of SVV infection including the development of rash, which lasted between 7 and 10 days. A representative RM infected with SVV BAC at 7

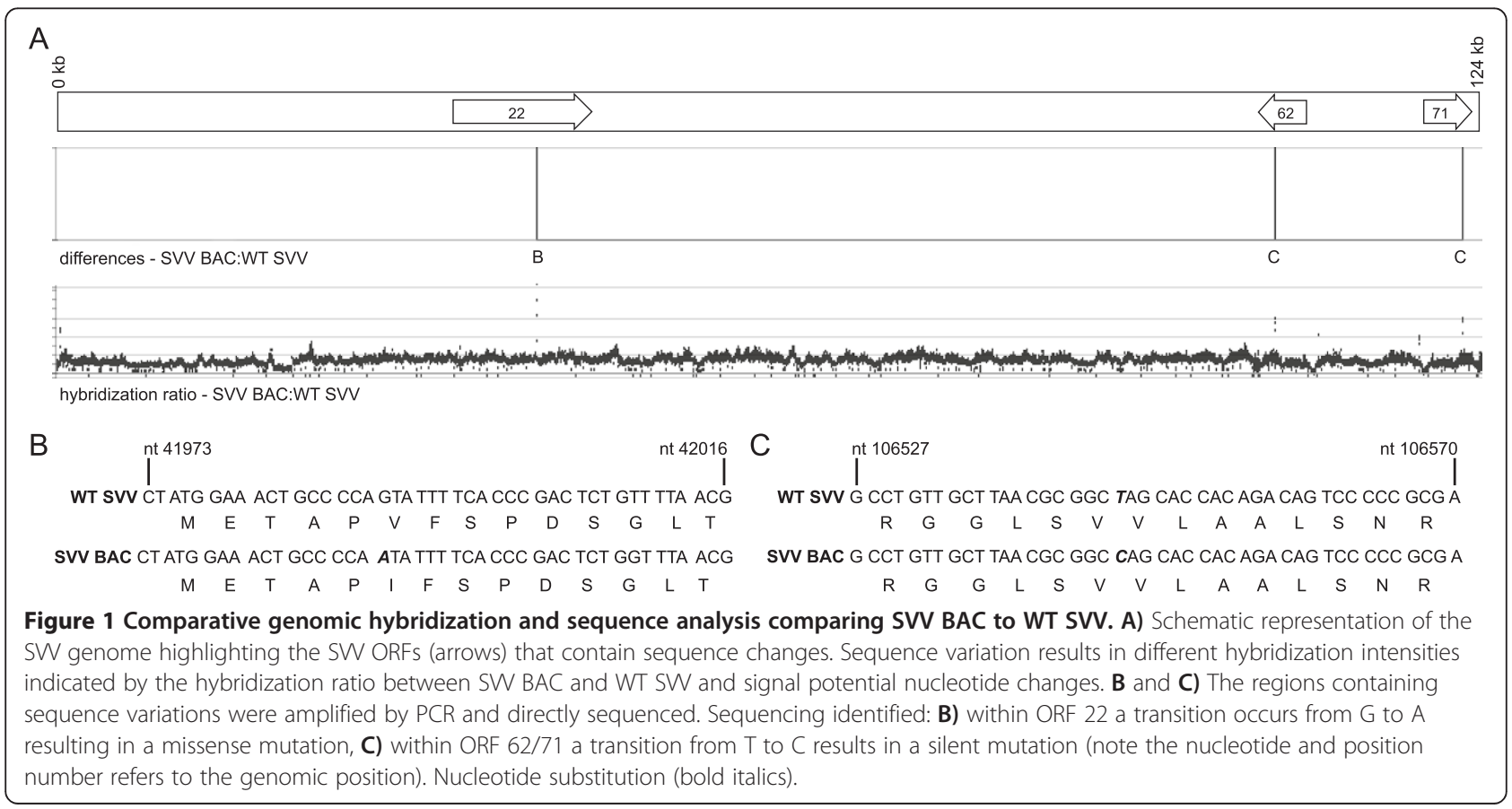


dpi is shown in Figure $2 \mathrm{~A}$ and a representative $\mathrm{RM}$ infected with WT SVV at $7 \mathrm{dpi}$ is shown in Figure 2B. A lesion area was biopsied at $10 \mathrm{dpi}$ and viral loads were measured by quantitative real-time PCR (Figure 2C). By $10 \mathrm{dpi}$, we were able to detect viral DNA in all RMs except RMs 28553 and 28621 infected with WT SVV. SVV viral loads were also measured by quantitative real-time PCR in BAL cells and whole blood samples. BAL cell viral loads peaked at $3 \mathrm{dpi}$ in both SVV BAC and WT SVV infected RMs then decreased to levels near or below our limit of detection by 63 dpi (Figure 2C). SVV viral loads in whole blood are significantly lower than in BAL cells, though we were able to detect SVV DNA in whole blood between 3 and 14 dpi in RMs infected with SVV BAC or WT SVV and then viral loads decreased to levels near or below our limit of detection (Figure 2D). Therefore, the ability of SVV BAC to replicate in vivo was comparable to WT SVV.

\section{Cytokine and chemokine levels in BAL supernatant and plasma}

We measured the concentrations of multiple chemokines (Figure 3A), cytokines (Figure 3B), and growth factors (Figure $3 \mathrm{C}$ ) in BAL fluid and plasma (data not shown) by multiplex technology. In BAL fluid infection with either SVV BAC or WT SVV induced production of several key chemokines, including MCP-1 (recruits monocytes, memory T cells, DCs [14]), MDC (recruits monocytes, monocyte-derived DCs, and NK cells [15]), MIF (inflammatory and atherogenic leukocyte recruitment [16]), MIG (recruits T cells [17]), MIP-1 $\alpha$ (recruits and activates polymorphonuclear leukocytes [18]), MIP$1 \beta$ (recruits NK cells and monocytes [18]), I-TAC (recruits T cells [19]), and eotaxin (recruits eosinophils [20]) (Figure 3). Concentrations of these chemokines peaked at $7 \mathrm{dpi}$ and returned to baseline by $14 \mathrm{dpi}$.

Concentrations of pro-inflammatory cytokines IFN $\gamma$ (involved in anti-viral activities and differentiation of $\mathrm{T}$ helper 1 (Th1) subsets [21]), TNF $\alpha$ (systemic inflammation [22]), IL-6 (pro- and anti-inflammatory responses [23]), IL-2 (T cell proliferation and homeostasis [24]) and IL-15 (proliferation of T cells and NK cells [25]) peaked at $7 \mathrm{dpi}$. The concentrations of IL-1 receptor antagonist (RA), which prevents IL-1 from signaling through the IL-1R [26] and IL-10 (pleiotropic activities in inflammation and immune regulation [27]) peaked at 7 dpi. G-CSF, a pleiotropic cytokine, produced by endothelium, macrophages and other immune cells, which stimulates the survival, proliferation, differentiation, and function of neutrophils also peaked at 7 dpi [28]. Levels
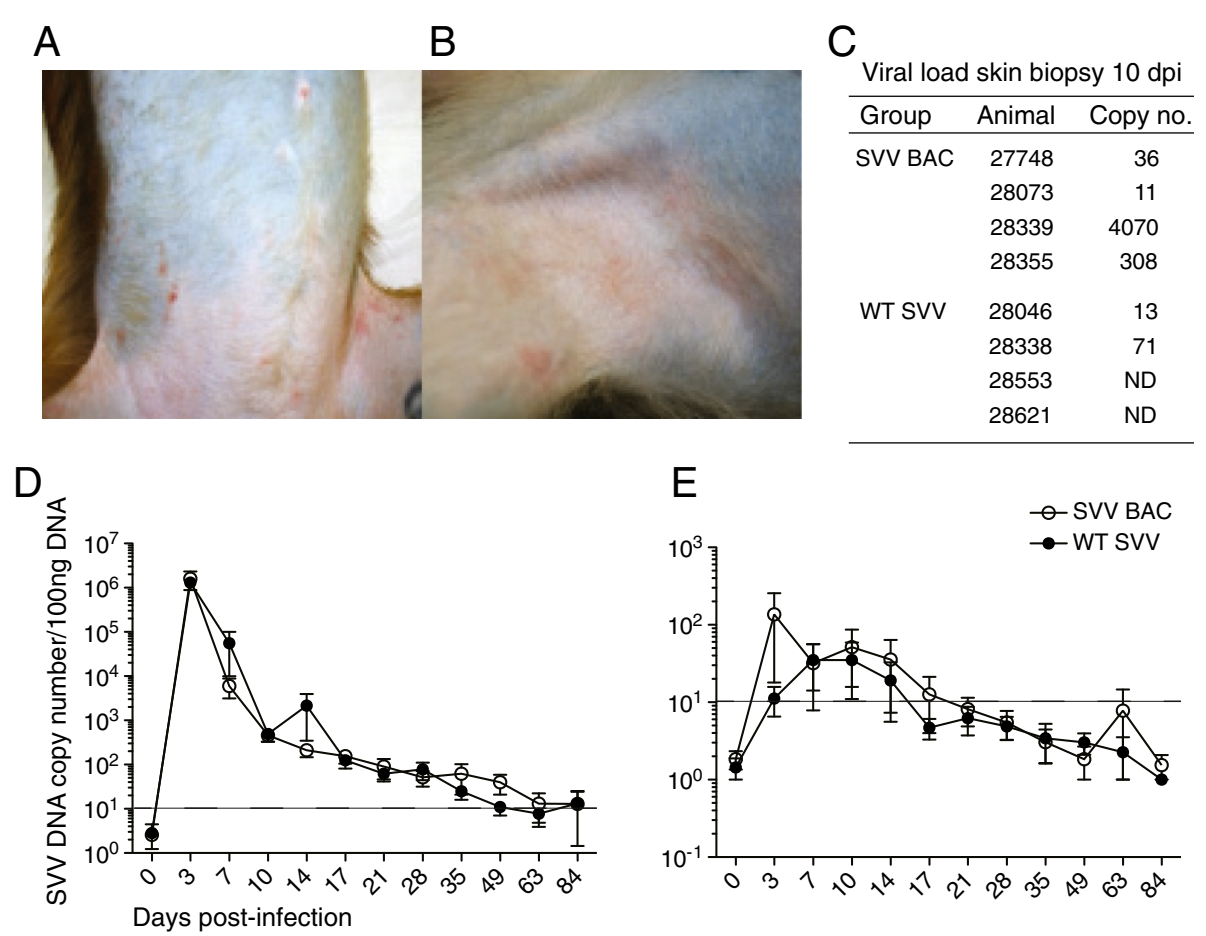

Figure 2 Varicella and viral load in SVV BAC and WT SVV infected RMs. A and B) Representative examples of varicella in WT SW and SW BAC infected RMs. (A) SW BAC infected RM on the trunk region and (B) WT SW infected RM on the axillary region at 7dpi. SW DNA viral load in C) skin biopsy at 10 dpi measured by quantitative PCR using primers and probe specific for SW ORF21. Average copy number per microgram of DNA. SW DNA viral load in D) BAL and E) whole blood was measured by quantitative PCR using primers and probe specific for SW ORF21 from SW BAC infected RMs (open circle) and WT SW infected RMs (closed circle). Dashed line indicates limit of detection. 


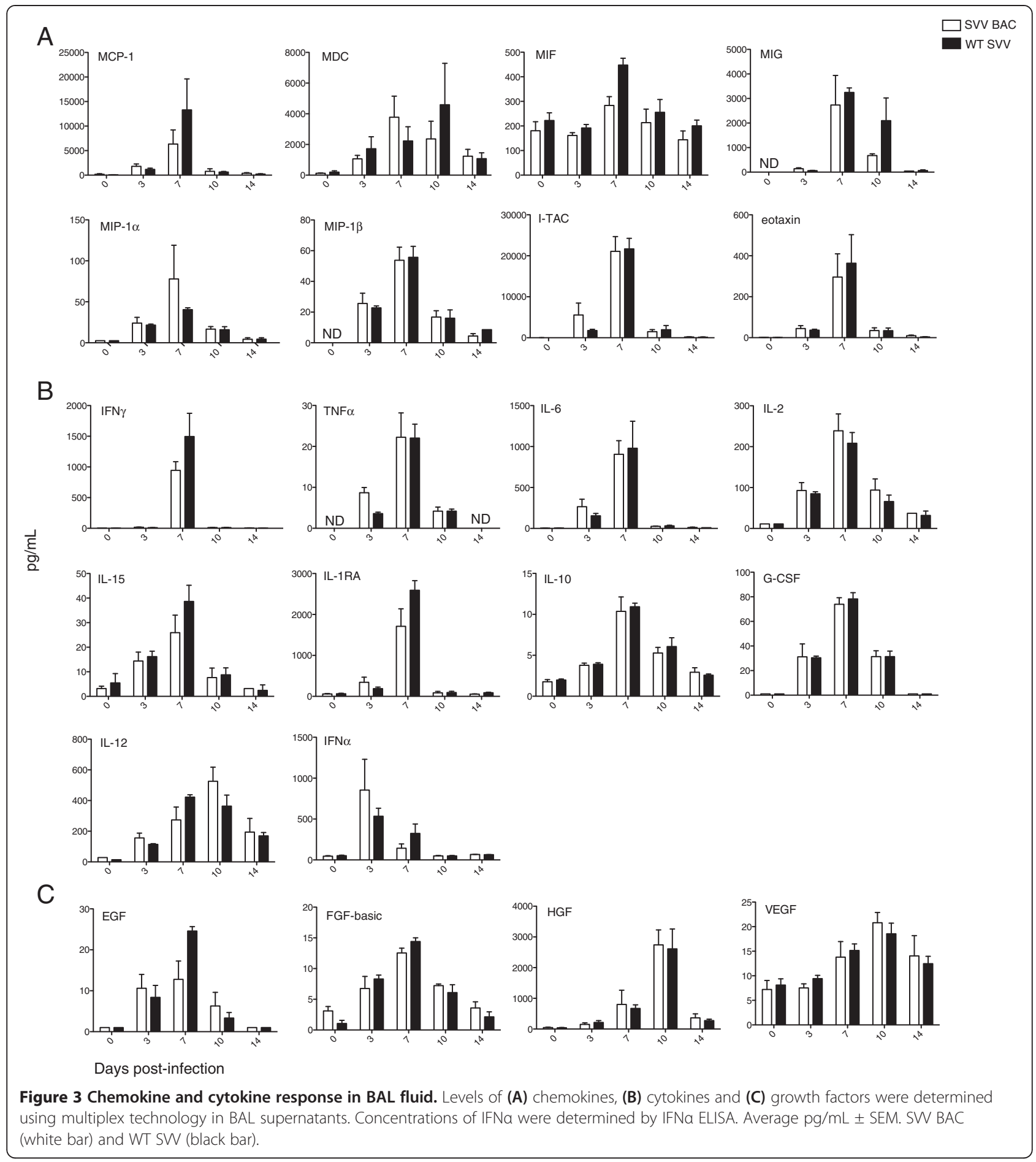

of IL-12, important in the differentiation of naïve $\mathrm{T}$ cells into CD4 Th1 cells [29], peaked at 10 dpi. Lastly, the concentration of IFN $\alpha$, a type I interferon that is important in anti-viral immunity, peaked at $3 \mathrm{dpi}$.

Growth factors EGF (stimulates cell growth, proliferation and differentiation [30]), and FGF-basic (multifunctional protein involved in angiogenesis and wound healing
[31]) concentrations in BAL fluid peaked at $7 \mathrm{dpi}$. While levels of growth factors HGF (regulates cell growth, cell motility, and morphogenesis, and acts primarily upon epithelial and endothelial cells [32]) and VEGF (stimulates vasculogenesis and angiogenesis [33]) peaked at $10 \mathrm{dpi}$.

Concentrations of IL-1 $\beta$, IL-4, IL-5, IL-8, IL-17, GMCSF and RANTES (CCL5) and in BAL fluid were below 
our limit of detection (data not shown). We did not detect any significant differences in concentrations of chemokines, cytokines or growth factors between RMs infected with SVV BAC or WT SVV.

We also measured the concentration of the above chemokines, cytokines, and growth factors in plasma (data not shown). However, we were only able to detect changes in the levels of IFN $\gamma$, which peaked at $7 \mathrm{dpi}$ and returned to baseline by $10 \mathrm{dpi}$ and no significant differences were detected between cohorts.

\section{B cell and antibody response to SVV BAC}

We compared the magnitude and kinetics of the $B$ cell response as well as the generation of SVVspecific IgG antibody titers post-infection in RMs infected with SVV BAC or WT SVV. The expansion of $B$ cells is measured based on expression of Ki67, a nuclear protein involved in DNA replication [34] by flow cytometry. SVV infection induces the proliferation of $B$ cells indicated by an increase in the frequency of Ki67 positive cells on days 7 to 14 compared to day 0 in marginal zone (MZ)-like (CD27 ${ }^{+}$ $\mathrm{IgD}^{+}$) and memory $\left(\mathrm{CD}_{27}{ }^{+} \mathrm{IgD}^{-}\right) \mathrm{B}$ cells, in BAL cells (Figure 4A) and PBMC (Figure 4B). In BAL cells and PBMC we measured similar proliferation of both subsets of $\mathrm{B}$ cells and no statistical differences in RMs infected with either SVV BAC or WT SVV except for at 10 dpi in BAL cells of WT SVV infected RMs we detected higher proliferation of MZ-like B cells $(\mathrm{p}<0.05)$ compared to SVV BAC. We also measured SVV-specific IgG (Figure 4C) antibody endpoint titers in plasma using standard ELISA. The kinetics of IgG production were comparable during SVV BAC and WT SVV infection of RMs, the titers peaked around day 14 post-infection and remained stable until necropsy.
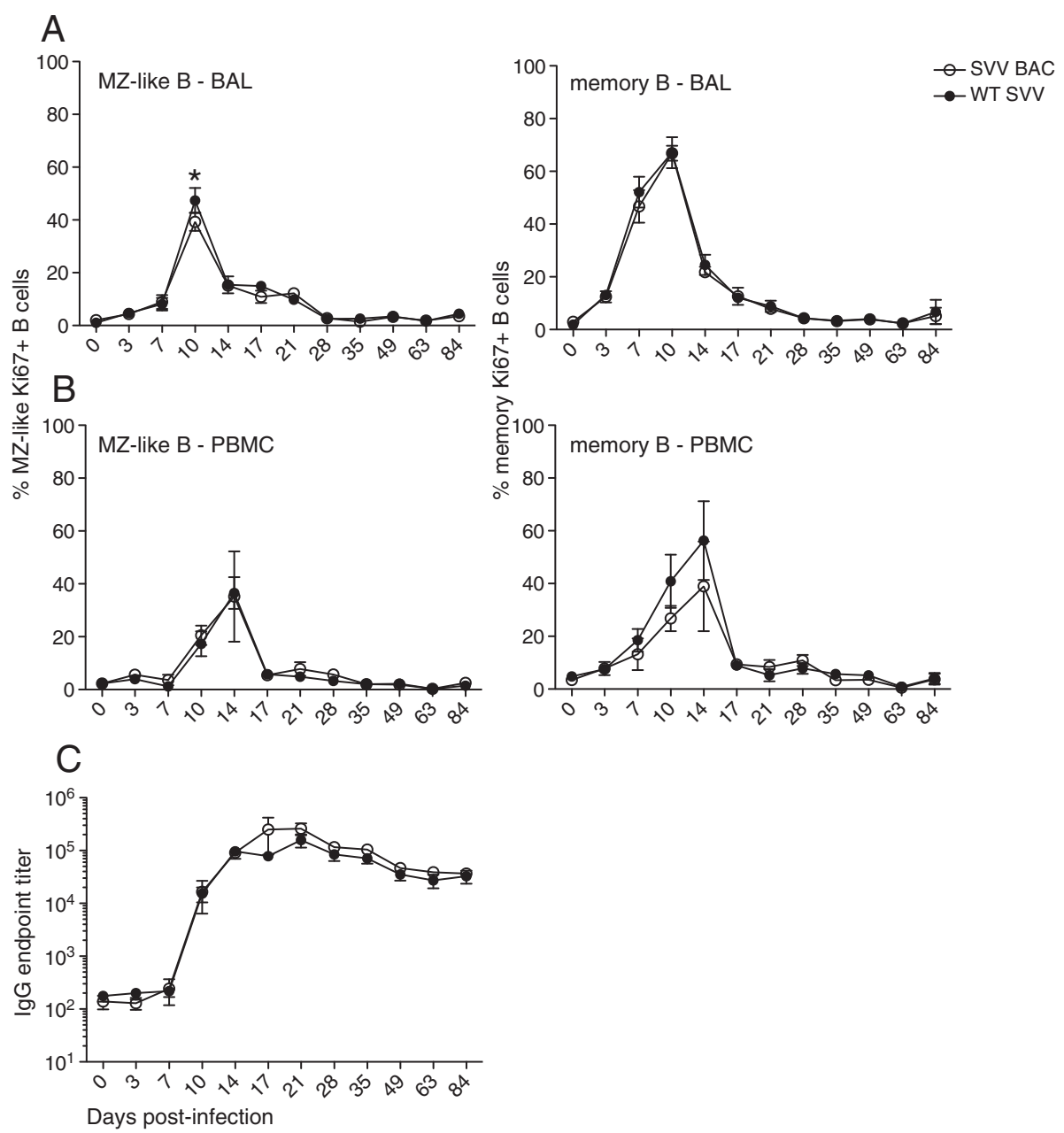

Figure 4 B cell proliferation and IgG production. The frequency of proliferating in MZ-like and memory B cell subsets was measured using flow cytometry based on the expression of Ki67 in (A) BAL and (B) PBMC. SW-specific (C) lgG antibody endpoint titers were measured by standard ELISA. SW BAC (open circle) and WT SW (closed circle). Average \pm SEM * $p<0.05$. 


\section{T cell response to SVV BAC}

Naïve $T$ cells following antigen encounter become activated, proliferate, and differentiate into central memory $\left(\mathrm{CM}, \mathrm{CD} 28^{+} \mathrm{CD} 95^{+}\right)$and effector memory (EM, CD28 $\mathrm{CD}^{+} 5^{+}$) $\mathrm{T}$ cells. We compared the kinetics and magnitude of the $\mathrm{T}$ cell response by measuring the frequency of Ki67 positive CM and EM T cells subsets in BAL cells (Figure 5A) and PBMC (Figure 5B) in SVV BAC or WT SVV infected RMs. SVV BAC and WT SVV infection induced strong $\mathrm{T}$ cell proliferation in BAL cells as shown by an increase in Ki67 positive T cells from days 7 to 17 post-infection. Within PBMC, T cell proliferation was detected in CD4 EM, CD8 CM and CD8 EM subsets but the magnitude was significantly reduced compared to the proliferation observed in BAL cells. Similarly though, the magnitude and kinetics was comparable in RMs infected with SVV BAC and WT SVV.

Additionally, we determined the frequency of SVVspecific $\mathrm{T}$ cells within CD4 and CD8 $\mathrm{T}$ cell populations by measuring the combined number of IFN $\gamma^{-}$, TNF $\alpha-$ and, IFN $\gamma / \mathrm{TNF} \alpha$-producing cells following stimulation with either SVV lysate or a SVV overlapping peptide pool covering ORFs 4, 31, 61 and 63 using intracellular cytokine staining (ICS). We stimulated both BAL cells (Figure 6A-D) and PBMC (Figure 6E,F) isolated from infected RMs at different dpi. Within BAL cells of SVV

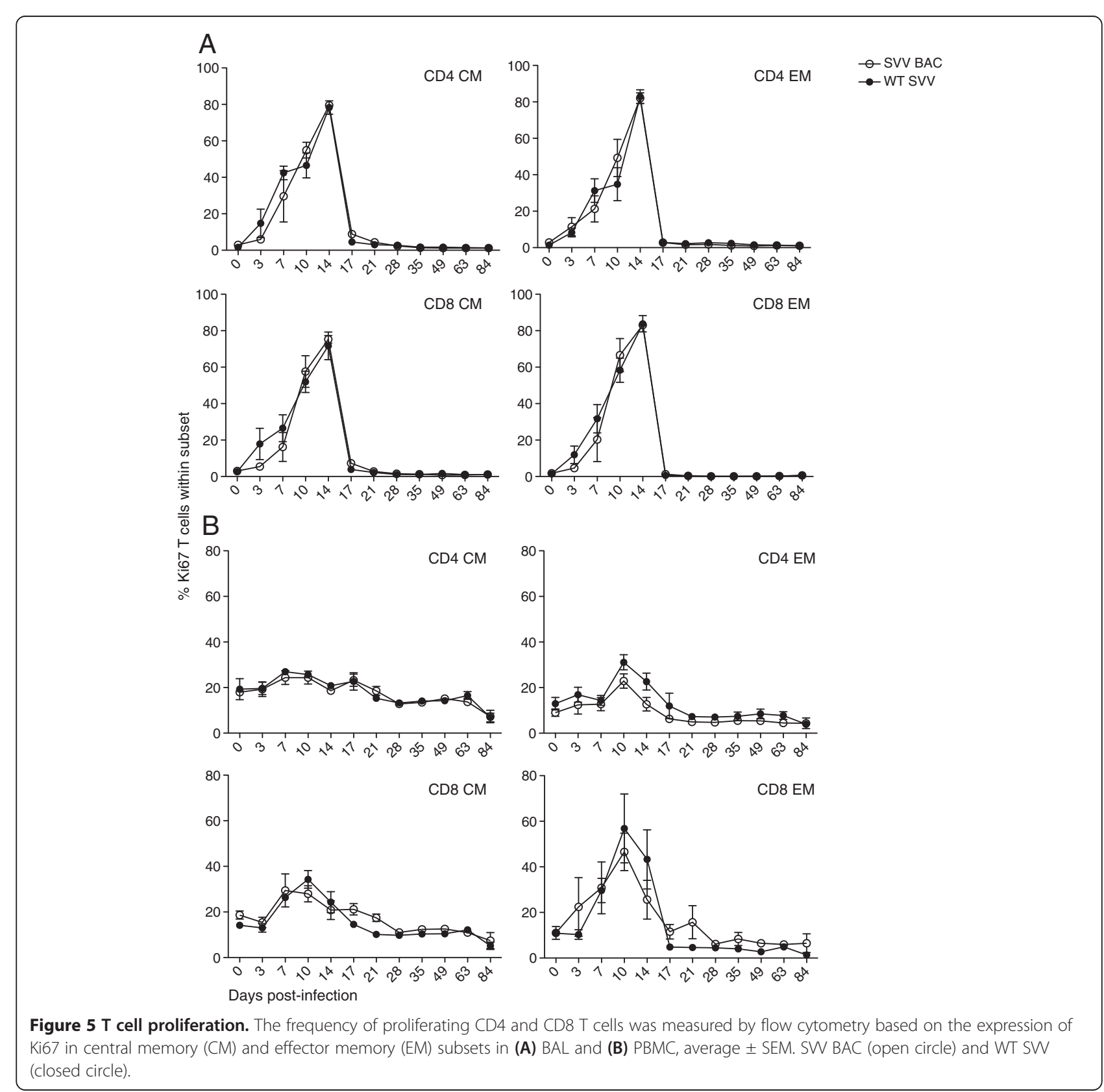



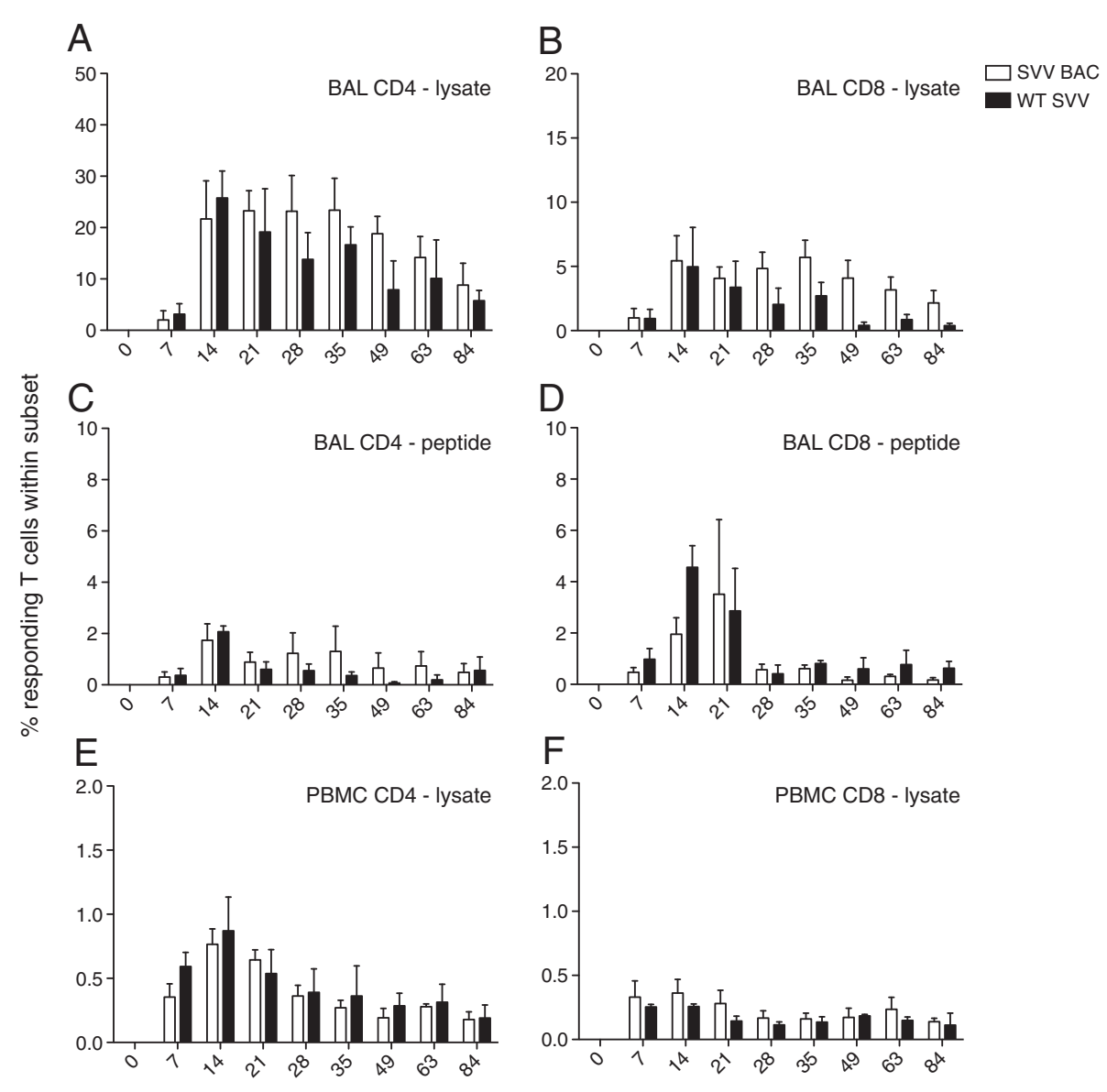

Days post-infection

Figure 6 The frequency of responding SVV-specific T cells. The frequency of SW-specific T cells in (A-D) BAL and (E and F) PBMC producing IFNY, TNFa and IFNY/TNFa was measured by intracellular cytokine staining following stimulation with either (A, B, E and F) SW lysate or (C and D) overlapping peptide pool (SW ORFs 4, 31, 61 and 63), average \pm SEM. SW BAC (white bar) and WT SW (black bar).

BAC or WT SVV infected RMs, SVV-specific CD4 and CD8 $\mathrm{T}$ cells were detected $7 \mathrm{dpi}$, their frequency peaked between 14 and $21 \mathrm{dpi}$ and declined to a memory set point. There were no statistically significant differences between animals infected with SVV BAC or WT SVV, and both cohorts did not produce a measurable CD4 or
CD8 response in PBMCs following stimulation with overlapping viral peptide pools (data not shown).

\section{SVV viral load in sensory ganglia}

SVV DNA viral loads in sensory ganglia were measured by quantitative PCR (Table 1). The viral loads reported

Table 1 SVV viral load in sensory ganglia

\begin{tabular}{|c|c|c|c|c|c|}
\hline SVV BAC animal ID & Sample & Copy no. ${ }^{a}$ & WT SVV animal ID & Sample & Copy no. ${ }^{a}$ \\
\hline \multirow[t]{4}{*}{28339} & TG & 576 & 28046 & TG & 14 \\
\hline & DRG-C & ND & & DRG-C & ND \\
\hline & DRG-T & 1526 & & DRG-T & ND \\
\hline & DRG-L/S & 67 & & DRG-L/S & ND \\
\hline \multirow[t]{4}{*}{28355} & TG & ND & 28553 & TG & 41 \\
\hline & DRG-C & 17 & & DRG-C & ND \\
\hline & DRG-T & ND & & DRG-T & ND \\
\hline & DRG-L/S & ND & & DRG-L/S & ND \\
\hline
\end{tabular}

a average copy number per ug of DNA.

DRG-C, cervical dorsal root ganglia; DRG-T, thoracic dorsal root ganglia; DRG-L/S, lumbar/sacral dorsal root ganglia; ND, not detected. 
in Table 1 reflect SVV genome copy numbers in a portion of the ganglia and are therefore not representative of the entire organ. Four of the RMs went on to further studies therefore Table 1 shows the latent viral loads for two animals from each cohort. We detected SVV DNA within at least one sensory ganglia of each RM infected with SVV BAC and WT SVV indicating that the SVV BAC, like WT SVV is able to traffic to the sensory ganglia, the site of SVV latency.

\section{Discussion}

Cloning viral genomes as bacterial artificial chromosomes (BAC) is an efficient tool to manipulate the viral genome facilitating the study of viral genes in vitro and in vivo. BACs were constructed for the VZV parental Oka virus and the vaccine Oka virus [35-38]. All VZV ORFs have been deleted using BACs or cosmids and tested in cell culture providing valuable information on which VZV genes are essential for viral replication in vitro (reviewed in $[39,40]$ ). Subsequent in vivo analysis of VZV mutant viruses using the SCID-hu mouse model showed that VZV ORFs 1,2 , and 3 are dispensable for viral replication [38], whereas VZV ORF23, which was dispensable for replication in culture, was found to be required for replication in human skin xenografts [41]. Similarly, VZV deleted for ORF7 replicated in MeWo cells but in vivo ORF7 was shown to be important for neuroinvasion $[40,42]$. Lastly, mutating the furin recognition site of VZV ORF31 (gB), an essential viral protein, did not affect VZV replication in vitro but attenuated viral replication in vivo [43]. Studying VZV mutant viruses in the SCID-hu mouse model has provided valuable insight into VZV biology, further evaluation of the role of different VZV ORFs in pathogenesis and the immune response in vivo is hampered by the fact that VZV is an obligate human pathogen. SVV is a simian homo$\log$ of VZV that causes varicella and zoster in nonhuman primates [4-6].

Recently, the SVV genome was cloned as a BAC and SVV virus generated using the SVV BAC genetic system was found to be similar to WT SVV in vitro [11]. Mutagenesis of SVV ORF10 showed that SVV ORF10 is nonessential for replication in vitro [11]. Additionally, generation of a SVV ORF 63/70 mutant demonstrated impaired growth in Vero cells [44]. In the present study we further the analysis of SVV BAC by infecting rhesus macaques and investigating the pathogenesis of SVV BAC compared to WT SVV in vivo. The combination of the rhesus macaque animal model and the SVV BAC will provide a robust tool to examine viral ORFs important for pathogenesis and help to target VZV ORFs that will improve vaccine efficacy.

To compare the genome of the SVV BAC to WT SVV we utilized comparative genomic hybridization. CGH analysis was employed as a cost-effective and accurate strategy to analyze genomic DNA from multiple viruses. This technique is sensitive enough to detect single base changes in addition to insertions, deletion or rearrangements in the genome [45-47]. We sequenced two sites in the SVV BAC genome that displayed variations in hybridization when compared to WT SVV. In ORF22 a point mutation at nucleotide 41990 was found producing an amino acid change from valine to isoleucine. SVV and VZV ORF22 are putative tegument proteins based on homology to HSV-1 UL36 [48]. UL36 is a HSV-1 late gene and the HSV-1 virion contains 100-150 copies of UL36 [49,50]. UL36 is essential to HSV-1 replication and the phenotype of a null mutant virus showed accumulation of capsids containing cytosolic DNA that did not mature into enveloped virions [51-53]. Though, the evidence suggests that the amino acid change in SVV BAC does not constitute a significant change in the protein. In vitro SVV BAC displays similar plaque size and replication kinetics in Vero cell monolayers as compared to WT SVV [11]. Our data in vivo shows that SVV BAC displays similar replication kinetics, immune response and establishment of latency compared to WT SVV. Potentially the position of the amino acid change or that the change is a nonpolar side chain to a nonpolar side change allows for WT behavior.

The replication kinetics of SVV BAC in the bronchoalveolar lavage cells (the site of virus inoculation) and in the peripheral blood was statistically similar to WT SVV. Related, the spread of varicella rash was also similar between cohorts and lasted between 7 and 10 days post-infection. In Figure $2 \mathrm{~A}$ and $\mathrm{B}$ we show pictures of two RMs infected with SVV BAC or WT SVV, which showed representative rash and also illustrates the variation in rash spread we see in our animals. In two animals infected with WT SVV, we did not detect viral DNA in the skin lesions but due to the nature of the skin punch biopsies as well as the timing of the rash, which varies between animals usually between 7 to 10 days post-infection, we may not have obtained a lesion spot with detectable viral DNA.

We also followed the immune response to SVV BAC during acute infection and found SVV BAC to elicit a parallel immune response in vivo. We analyzed the proliferation kinetics of antigen experienced B cells, the production of SVV-specific IgG antibodies as well as the proliferation kinetics of memory $\mathrm{T}$ cells and the IFN $\gamma /$ TNFo response of SVV-specific CD4 and CD8 T cells, and each parameter was analogous. We did measure a statistical difference in MZ-like B cells at $10 \mathrm{dpi}$ in the BAL where RMs infected with WT SVV displayed a higher peak percentage of Ki67 positive cells. However, this difference did not translate to a higher antibody titer in the WT SVV infected RMs. This difference could be 
due to the small sample size $(n=4)$ and the outbred nature of rhesus macaques.

Infection with SVV BAC also resulted in a comparable upregulation of chemokines, cytokines, and growth factors during the early stages of acute infection in the lungs compared to RMs infected with WT SVV. Peak levels of IFN $\alpha$, an important antiviral cytokine, occurred at $3 \mathrm{dpi}$, which corresponds to peak viral loads in the lungs also at $3 \mathrm{dpi}$. Type I interferons are early immune effectors and are important cytokines during initial infection to limit viral replication and spread, including herpesviruses [54-56]. The concentrations of several $\mathrm{T}$ cell-recruiting chemokines (MCP-1, MIG, I-TAC) peaked 7 days prior to the observed peak in proliferating T cells in BAL samples. We also detected increased concentrations of TNF $\alpha$ ( $3 \mathrm{dpi})$ and IFNY $(7 \mathrm{dpi})$, which is indicative of Th1 immune responses and correlates with an increase in CD4 CM in BAL samples. Peak concentrations of IL-2 were detected 7 days prior $(7 \mathrm{dpi})$ to the peak proliferation of CD4 and CD8 T cells (14 dpi). Levels of IL-12, which play a role in enhancing cytotoxic function of CD8 T cells, peaked 4 days before peak proliferation of CD8 T cells in BAL samples. Interestingly, we also detected an increase in growth factors in the BAL fluid, which peaked from 7 to 10 days postinfection. Many of these growth factors are involved in wound healing and might represent a response to tissue injury induced by SVV replication within the lungs (site of inoculation).

Lastly we found that both SVV BAC and WT SVV established latency within the sensory ganglia. Viral DNA was detected in at least one ganglion from each RM measured by quantitative PCR. In summary, SVV BAC is as pathogenic in vivo as WT SVV. Future studies will utilize the SVV BAC genetic system to generate knockout viruses to help characterize the role of SVV genes in acute infection, the establishment and maintenance of latency, and reactivation in vivo, a critical step in the understanding of viral factors that impact VZV pathogenesis and the immune response to VZV.

\section{Materials and methods}

\section{Cells and viruses}

Bacterial artificial chromosome (BAC)-derived SVV was generated from self-excisable pSVV-BAC resulting in complete excision of plasmid sequences from the virus genome [11]. Wild-type (WT) simian varicella virus (SVV, Cercopithecine herpesvirus 9) and SVV BAC were propagated as previously described, briefly Vero cells maintained in Eagle's minimal essential medium (EMEM) supplemented with 5\% newborn bovine serum, penicillin and streptomycin, WT SVV and SVV BAC infected Veros were harvested by scraping and frozen in Vero media supplemented with $10 \%$ dimethyl sulfoxide
(DMSO) [11]. Virus stocks were titered by plaque assay on primary rhesus fibroblasts maintained in Dulbecco's modified Eagle's Medium (DMEM) supplemented with $10 \%$ fetal bovine serum and penicillin, streptomycin and L-glutamine. WT SVV cell lysate was obtained by scraping infected primary rhesus fibroblasts at the height of CPE followed by centrifugation and sonication using 7 pulses of 70-80 Watts (Sonicator 3000, Misonix Inc., Farmingdale NY) and frozen at $-80^{\circ} \mathrm{C}$.

\section{Animals and sample collection}

All rhesus macaques were housed at the Oregon National Primate Research Center and were handled in accordance with good animal practices as defined by the Office of Laboratory Animal Welfare. Animal work was approved by the Oregon National Primate Research Center Institutional Animal Care and Use Committee. Rhesus macaques (RM, Macaca mulatta) were SVV seronegative prior to infection measured by ELISA. RMs $(\mathrm{n}=4$ per group) were infected intrabronchially with $4 \times 10^{5}$ PFU WT SVV or SVV BAC infected Veros. Peripheral blood mononuclear cells (PBMC) and bronchoalveolar lavage (BAL) cells were collected from rhesus macaques as previously described [5]. Animals were euthanized at 84 to 86 days post-infection. Sensory ganglia: trigeminal ganglia (TG), cervical, thoracic and lumbarsacral dorsal root ganglia (DRG-C, DRG-T, and DRG$\mathrm{L} / \mathrm{S}$ respectively) were divided, flash frozen and stored at $-80^{\circ} \mathrm{C}$ until analysis.

\section{Comparative genome analysis of SVV BAC and SVV WT DNA}

A microarray hybridization-based method was used to compare SVVDORF61 genomic DNA (test) to WT SVV (reference) DNA provided by NimbleGen Systems, Inc. (CGS 385K Mutation Mapping array Phase 1, Madison WI). Design of the microarray used published sequence data for the Delta herpesvirus strain of SVV (NC_00 2686, [8]). The oligonucleotides were $29-39 \mathrm{bp}$ in length and tiled throughout the genome every 7-8 bases on both forward and reverse strands. Viral DNA was isolated from nucleocapsids purified from SVV-infected Vero cells as previously described [7]. Hybridization data was analyzed using SignalMap software (NimbleGen Systems, Inc., Madison WI). The identified mismatches were directly sequenced from PCR products obtained from amplifying the surrounding sequence. The primers employed for Figure 1 include: (B) primer 1, 5' -CCATA TGTACCAACGGGAACA-3' and primer 2, 5' -AAGCA TGCATTTTCGATTGGA-3'; (C) primer 1, 5'-GCCTG GAGCCCAGATATTCGA-3' and primer 2, 5' -ACGGT GTGCGTGGATGCATCA-3'. 
DNA extraction and quantitative real-time PCR (qPCR)

DNA was extracted from heparinized whole blood (WB), BAL cells, and portions of frozen ganglia using Archive Pure DNA Cell/Tissue Kit (5 PRIME, Gaithersburg MD) according to the manufacturer's protocol. SVV DNA viral loads in WB, BAL cells and sensory ganglia were measured by qPCR using Maxima Probe/ROX qPCR Master Mix (2X) (Fermentas, Glen Burnie MD) and primers/Taqman probe specific for SVV ORF21. Following an initial 10 minute $95^{\circ} \mathrm{C}$ step, 40 cycles of $15 \mathrm{sec}$ at $95^{\circ} \mathrm{C}$ and 1 minute at $60^{\circ} \mathrm{C}$ were completed using StepOnePlus (Life Technologies, Carlsbad CA). SVV BAC DNA was used as quantification standards [11].

\section{Cytokine analysis}

Plasma and BAL supernatant samples (stored at $-80^{\circ} \mathrm{C}$ ) were thawed and analyzed using Cytokine Monkey Magnetic 28-plex panel as per the manufacturer's instructions (Life Technologies). IFN $\alpha$ levels were measured using Cynomolgus/Rhesus IFNa Serum ELISA Kit according to the manufacturer's instructions (PBL Interferon Source, Piscataway NJ). Samples were run in duplicate. Values below the limit of detection were designated as ND, or not detected.

\section{Enzyme-linked immunosorbent assay (ELISA)}

ELISA plates were coated with SVV lysate overnight at $4^{\circ} \mathrm{C}$, blocked with $5 \%$ milk in wash buffer $(0.05 \%$ Tween in PBS) for $1 \mathrm{~h}$ at room-temperature (RT), washed three times with wash buffer, and incubated with heatinactivated $\left(55^{\circ} \mathrm{C}, 30 \mathrm{~min}\right)$ plasma samples in 3-fold dilutions in duplicate for $1 \mathrm{~h}$. After washing three times with wash buffer, horseradish peroxidase (HRP)-conjugated anti-rhesus IgG (Nordic Immunology, Netherlands) was added for $1 \mathrm{~h}$, followed by addition of chromagen 0 phenylenediamine $2 \mathrm{HCl}$ (OPD) (Sigma, St Louis MO) substrate for 20 minutes to allow detection and quantitation of bound antibody molecules. The reaction was stopped with the addition of $1 \mathrm{M} \mathrm{HCl}$. The optical density was measured at $490 \mathrm{~nm}$ using an ELISA plate reader (SpectraMax 190, Molecular Devices, Sunnyvale CA). Endpoint IgG titers were calculated using log-log transformation of the linear portion of the curve with 0.1 optical density (OD) units as the cut-off. Titers were standardized using a positive control sample included with each assay.

\section{Measurement of $\mathrm{T}$ cell and B cell frequency and proliferation}

BAL cells and PBMC were surface stained with antibodies against 1) CD4 (eBioscience, San Diego CA), CD8 $\beta$ (Beckman Coulter), CD28, and CD95 (BioLegend, San Diego CA) to delineate the naive $\left(\mathrm{CD} 28^{+} \mathrm{CD} 95^{-}\right)$, central memory $\left(\mathrm{CD} 28^{+} \mathrm{CD} 95^{+}\right)$, and effector memory
$\left(\mathrm{CD} 28^{-} \mathrm{CD}^{+} 5^{+}\right) \mathrm{T}$ cell subsets; 2) CD20 (Beckman Coulter, Brea CA), IgD (Southern Biotech, Birmingham $\mathrm{AL}$ ), and $\mathrm{CD} 27$ (BioLegend) to delineate naïve $\left(\mathrm{CD} 20^{+}\right.$ $\left.\operatorname{IgD}^{+} \mathrm{CD} 27^{-}\right)$, marginal zone-like (MZ-like) $\left(\mathrm{CD}^{2} \mathrm{O}^{+} \mathrm{IgD}^{+}\right.$ $\left.\mathrm{CD} 27^{+}\right)$, and memory $\left(\mathrm{CD} 20^{+} \mathrm{IgD}^{-} \mathrm{CD} 27^{+}\right) \mathrm{B}$ cell subsets. Cells were fixed and permeabilized according to manufacturer recommendations (BioLegend) before the addition of Ki67-specific antibody (BD Biosciences, San Jose, CA). The samples were analyzed using the LSRII instrument (Beckton, Dickinson and Company, San Jose CA) and FlowJo software (TreeStar, Ashland OR).

\section{Intracellular cytokine staining}

BAL cells and PBMC were stimulated with SVV lysate $(1 \mu \mathrm{g})$ or SVV overlapping peptide pool containing open reading frames (ORFs) 4, 31, 61 and 63 for $1 \mathrm{~h}$ followed by addition of Brefeldin A (Sigma, St Louis MO) to block cytokine export for $14 \mathrm{~h}$. After stimulation cells were surface stained with antibodies against $\mathrm{CD} 4$, and $\mathrm{CD} 8 \beta$, as described above. Samples were fixed, permeabilized (BioLegend) and dual-stained using antibodies against IFNY (eBioscience) and TNF $\alpha$ (eBioscience). Samples were analyzed using the LSRII instrument and Flowjo software.

\section{Statistical analysis}

Statistical analysis and graphing was conducted with GraphPad Prism software (GraphPad Software Inc., La Jolla CA). Significance values for Figures 2, 3, 4, 5, and 6 utilized repeated measures of ANOVA with the Bonferroni post-test to explore differences between groups (SVV BAC and WT SVV) at each time-point.

\section{Competing interests}

The authors declared that they have no competing interests.

\section{Authors' contributions}

Study design: IM and CM; data collection: CM, JD, KH, FE, NA; data interpretation and manuscript preparation: IM and CM. WG provided SW BAC and WT SW. All authors read and approved the final manuscript.

\section{Acknowledgements}

We would like to thank the Division of Animal Resources (DAR) at the Oregon National Primate Research Center for expert animal care, especially Drs. Anne Lewis and Lois Colgin for conducting the necropsies and collecting tissues; Alfred Legasse, Miranda Fischer and Shannon Planer for collection of blood and BAL samples. This work was supported by American Heart Association career development grant 0930234N, NIH R01AG037042, 2T32Al007472-16, NIH 8P51 OD011092-53 and the Brookdale Foundation. The funding sources had no role in study design, data collection and analysis, decision to publish, or preparation of the manuscript.

\section{Author details}

'Vaccine and Gene Therapy Institute, Oregon National Primate Research Center, Beaverton, OR 97006, USA. ${ }^{2}$ Molecular Microbiology and Immunology Department, Oregon National Primate Research Center, Beaverton, OR 97006, USA. ${ }^{3}$ Division of Pathobiology and Immunology, Oregon National Primate Research Center, Beaverton, OR 97006, USA. ${ }^{4}$ Division of Biomedical Sciences, University of California-Riverside, Riverside, CA 92508, USA. ${ }^{5}$ Department of Microbiology and Immunology, University of Arkansas, Little Rock, AK 72205, USA. ${ }^{6}$ School of Medicine, University of California-Riverside, 900 University Avenue, Riverside, CA 92521, USA. 
Received: 10 July 2013 Accepted: 3 September 2013

Published: 8 September 2013

\section{References}

1. Oxman MN, Levin MJ: Vaccination against herpes zoster and postherpetic neuralgia. J Infect Dis 2008, 197(Suppl 2):S228-S236.

2. Oxman $\mathrm{MN}$, et al: $\mathrm{A}$ vaccine to prevent herpes zoster and postherpetic neuralgia in older adults. N Engl J Med 2005, 352(22):2271-2284.

3. Clarkson MJ, Thorpe E, McCarthy K: A virus disease of captive vervet monkeys (Cercopithecus aethiops) caused by a new herpesvirus. Arch Gesamte Virusforsch 1967, 22(1):219-234.

4. Mahalingam $\mathrm{R}$, et al: Simian varicella virus DNA in dorsal root ganglia. Proc Natl Acad Sci USA 1991, 88(7):2750-2752.

5. Messaoudi l, et al: Simian varicella virus infection of rhesus macaques recapitulates essential features of varicella zoster virus infection in humans. PLOS Pathog 2009, 5(11):e1000657.

6. Fletcher TM 3rd, Gray WL: DNA sequence and genetic organization of the unique short (US) region of the simian varicella virus genome. Virology 1993, 193(2):762-773.

7. Gray WL, et al: The simian varicella virus and varicella zoster virus genomes are similar in size and structure. Virology 1992, 186(2):562-572.

8. Gray $W L$, et al: The DNA sequence of the simian varicella virus genome. Virology 2001, 284(1):123-130

9. Gray WL, Oakes JE: Simian varicella virus DNA shares homology with human varicella-zoster virus DNA. Virology 1984, 136(1):241-246.

10. Davison AJ, Scott JE: The complete DNA sequence of varicella-zoster virus. J Gen Virol 1986, 67(Pt 9):1759-1816.

11. Gray WL, et al: Cloning the simian varicella virus genome in E. coli as an infectious bacterial artificial chromosome. Arch Virol 2011, 156(5):739-746.

12. Gray $\mathrm{WL}$, et al: Simian varicella virus gene 61 encodes a viral transactivator but is non-essential for in vitro replication. Arch Virol 2007 152(3):553-563.

13. Meyer C, Dewane J, Kerns A, Haberthur K, Barron A, Park B, Messaoudi I: Age and immune status of rhesus macaques impact simian varicella virus gene expression in sensory ganglia. J Virol 2013, 87:8294-8306.

14. Deshmane SL, et al: Monocyte chemoattractant protein-1 (MCP-1): an overview. J Interferon Cytokine Res 2009, 29(6):313-326.

15. Yamashita $U$, Kuroda E: Regulation of macrophage-derived chemokine (MDC, CCL22) production. Crit Rev Immunol 2002, 22(2):105-114

16. Bernhagen J, et al: MIF is a noncognate ligand of CXC chemokine receptors in inflammatory and atherogenic cell recruitment. Nat Med 2007, 13(5):587-596.

17. Liu MT, et al: Expression of Mig (monokine induced by interferongamma) is important in T lymphocyte recruitment and host defense following viral infection of the central nervous system. J Immunol 2001, 166(3):1790-1795.

18. Maurer M, von Stebut E: Macrophage inflammatory protein-1. Int J Biochem Cell Biol 2004, 36(10):1882-1886.

19. Liu MT, et al: The T cell chemoattractant IFN-inducible protein 10 is essential in host defense against viral-induced neurologic disease. J Immunol 2000, 165(5):2327-2330.

20. Griffiths-Johnson DA, et al: The chemokine, eotaxin, activates guinea-pig eosinophils in vitro and causes their accumulation into the lung in vivo. Biochem Biophys Res Commun 1993, 197(3):1167-1172.

21. Boehm U, et al: Cellular responses to interferon-g. Annu Rev Immunol 1997, 15:749-795.

22. Esposito E, Cuzzocrea S: TNF-alpha as a therapeutic target in inflammatory diseases, ischemia-reperfusion injury and trauma. Curr Med Chem 2009, 16(24):3152-3167.

23. Hirano T, et al: Biological and clinical aspects of interleukin 6. Immunol Today 1990, 11(12):443-449.

24. Liao W, Lin JX, Leonard WJ: Interleukin-2 at the crossroads of effector responses, tolerance, and immunotherapy. Immunity 2013, 38(1):13-25

25. Fehniger TA, Caligiuri MA: Interleukin 15: biology and relevance to human disease. Blood 2001, 97(1):14-32.

26. Arend WP, et al: Interleukin-1 receptor antagonist: role in biology. Annu Rev Immunol 1998, 16:27-55.

27. Moore KW, et al: Interleukin-10. Annu Rev Immunol 1993, 11:165-190.

28. Welte $K$, et al: Purification and biochemical characterization of human pluripotent hematopoietic colony-stimulating factor. Proc Natl Acad Sci USA 1985, 82(5):1526-1530.
29. Scott P: IL-12: initiation cytokine for cell-mediated immunity. Science 1993, 260(5107):496-497.

30. Carpenter G, Cohen S: Epidermal growth factor. J Biol Chem 1990, 265(14):7709-7712.

31. Yu PJ, et al: Basic fibroblast growth factor (FGF-2): the high molecular weight forms come of age. J Cell Biochem 2007, 100(5):1100-1108.

32. Warn R: Growth factors. A scattering of factors. Curr Biol 1994, 4(11):1043-1045.

33. Byrne AM, Bouchier-Hayes DJ, Harmey $\mathrm{JH}$ : Angiogenic and cell survival functions of vascular endothelial growth factor (VEGF). J Cell Mol Med 2005, 9(4):777-794

34. Pitcher CJ, et al: Development and homeostasis of T cell memory in rhesus macaque. J Immunol 2002, 168(1):29-43.

35. Nagaike $K$, et al: Cloning of the varicella-zoster virus genome as an infectious bacterial artificial chromosome in Escherichia coli. Vaccine 2004, 22(29-30):4069-4074.

36. Tischer BK, et al: A self-excisable infectious bacterial artificial chromosome clone of varicella-zoster virus allows analysis of the essential tegument protein encoded by ORF9. J Virol 2007, 81(23):13200-13208.

37. Yoshii $\mathrm{H}$, et al: Cloning of full length genome of varicella-zoster virus vaccine strain into a bacterial artificial chromosome and reconstitution of infectious virus. Vaccine 2007, 25(27):5006-5012

38. Zhang Z, et al: Genetic analysis of varicella-zoster virus ORF0 to ORF4 by use of a novel luciferase bacterial artificial chromosome system. J Virol 2007, 81(17):9024-9033.

39. Cohen Jl: The varicella-zoster virus genome. Curr Top Microbiol Immunol 2010, 342:1-14.

40. Zhang Z, et al: Genome-wide mutagenesis reveals that ORF7 is a novel VZV skin-tropic factor. PLoS Pathog 2010, 6:e1000971.

41. Chaudhuri V, et al: Functions of Varicella-zoster virus ORF23 capsid protein in viral replication and the pathogenesis of skin infection. J Virol 2008, 82(20):10231-10246.

42. Selariu A, et al: ORF7 of varicella-zoster virus is a neurotropic factor. J Virol 2012, 86(16):8614-8624.

43. Oliver SL, et al: Mutagenesis of varicella-zoster virus glycoprotein B: putative fusion loop residues are essential for viral replication, and the furin cleavage motif contributes to pathogenesis in skin tissue in vivo. J Virol 2009, 83(15):7495-7506.

44. Brazeau $E$, et al: Simian varicella virus open reading frame $63 / 70$ expression is required for efficient virus replication in culture. J Neurovirol 2011, 17(3):274-280.

45. Estep RD, et al: Construction of an infectious rhesus rhadinovirus bacterial artificial chromosome for the analysis of Kaposi's sarcomaassociated herpesvirus-related disease development. J Virol 2007, 81(6):2957-2969.

46. Robinson BA, et al: Viral interferon regulatory factors decrease the induction of type I and type II interferon during rhesus macaque rhadinovirus infection. J Virol 2012, 86(4):2197-2211

47. Wong CW, et al: Tracking the evolution of the SARS coronavirus using high-throughput, high-density resequencing arrays. Genome Res 2004, 14(3):398-405.

48. Gray WL: Simian varicella virus: molecular virology. Curr Top Microbiol Immunol 2010, 342:291-308.

49. McNabb DS, Courtney RJ: Characterization of the large tegument protein (ICP1/2) of herpes simplex virus type 1. Virology 1992, 190(1):221-232.

50. Heine JW, et al: Proteins specified by herpes simplex virus. XII. The virion polypeptides of type 1 strains. J Virol 1974, 14(3):640-651.

51. Batterson W, Furlong D, Roizman B: Molecular genetics of herpes simplex virus. VIII. Further characterization of a temperature-sensitive mutant defective in release of viral DNA and in other stages of the viral reproductive cycle. J Virol 1983, 45(1):397-407.

52. Knipe DM, Ruyechan WT, Roizman B: Molecular genetics of herpes simplex virus. III. Fine mapping of a genetic locus determining resistance to phosphonoacetate by two methods of marker transfer. J Virol 1979, 29(2):698-704

53. Desai PJ: A null mutation in the UL36 gene of herpes simplex virus type 1 results in accumulation of unenveloped DNA-filled capsids in the cytoplasm of infected cells. J Virol 2000, 74(24):11608-11618.

54. Hendricks RL, et al: Endogenously produced interferon alpha protects mice from herpes simplex virus type 1 corneal disease. J Gen Virol 1991, 72(Pt 7):1601-1610. 
55. Mikloska Z, Cunningham AL: Alpha and gamma interferons inhibit herpes simplex virus type 1 infection and spread in epidermal cells after axonal transmission. J Virol 2001, 75(23):11821-11826.

56. Sainz B Jr, Halford WP: Alpha/Beta interferon and gamma interferon synergize to inhibit the replication of herpes simplex virus type 1. J Virol 2002, 76(22):11541-11550.

doi:10.1186/1743-422X-10-278

Cite this article as: Meyer et al.: Bacterial artificial chromosome derived simian varicella virus is pathogenic in vivo. Virology Journal 2013 10:278.

\section{Submit your next manuscript to BioMed Central and take full advantage of:}

- Convenient online submission

- Thorough peer review

- No space constraints or color figure charges

- Immediate publication on acceptance

- Inclusion in PubMed, CAS, Scopus and Google Scholar

- Research which is freely available for redistribution 